\title{
Impurity dominated thin film growth
}

F. G. Cougnon, A. Dulmaa, R. Dedoncker, R. Galbadrakh, and D. Depla

Citation: Appl. Phys. Lett. 112, 221903 (2018); doi: 10.1063/1.5021528

View online: https://doi.org/10.1063/1.5021528

View Table of Contents: http://aip.scitation.org/toc/apl/112/22

Published by the American Institute of Physics 


\title{
Impurity dominated thin film growth
}

\author{
F. G. Cougnon, ${ }^{1}$ A. Dulmaa, ${ }^{1}$ R. Dedoncker, ${ }^{1}$ R. Galbadrakh, ${ }^{2}$ and D. Depla ${ }^{1}$ \\ ${ }^{1}$ Department of Solid State Sciences, Ghent University, Krijgslaan 281(S1), 9000 Gent, Belgium \\ ${ }^{2}$ Department of Physics, National University of Mongolia, Ulaanbaatar 14201, Mongolia
}

(Received 5 January 2018; accepted 14 May 2018; published online 30 May 2018)

\begin{abstract}
Magnetron sputter deposition was applied to grow thin metal films in the presence of impurities. These impurities are ambient gas molecules and/or atoms from the residual gas present in the vacuum chamber. Seven materials were investigated: four single element metals (Al, $\mathrm{Ag}, \mathrm{Cu}$, and $\mathrm{Cr}$ ), two widely applied alloys $\left(\mathrm{Cu}_{55} \mathrm{Ni}_{45}\right.$ and $\left.\mathrm{Ni}_{90} \mathrm{Cr}_{10}\right)$, and one high entropy alloy $(\mathrm{CoCrCuFeNi})$. The thin films were analyzed using X-ray diffraction to determine the domain size, the film texture, and the lattice parameter. The same trend for all studied materials is observed. When the ratio between the impurity and metal flux towards the substrate is low, the domain size is not affected by the presence of the impurities. In this regime, the incorporation of the impurities affects the lattice parameter. At high flux ratios, the change of the domain size can be described by a power law with the exponent equal to $-1 / 2$ for all studied materials. A kinetic Monte Carlo code is used to demonstrate this observed trend. Published by AIP Publishing. https://doi.org/10.1063/1.5021528
\end{abstract}

Foreign atoms, or impurities, during physical vapor deposition can affect the film growth on several levels. A good overview is given by Barna and co-workers in a dedicated study on the growth of evaporated aluminum thin films in the presence of oxygen. ${ }^{1-6}$ They discuss the influence of oxygen as an impurity and describe a change in the film texture together with a decrease in grain size as a function of an increasing oxygen pressure. Similar effects were demonstrated by the same research group during the growth of TiN thin films, ${ }^{7}$ by Liu and Barmak for $\mathrm{W},{ }^{8}$ by Mahieu et al. for $\mathrm{Cr},{ }^{9}$ and by Riedl et al. for TiAlN. ${ }^{10}$ In the latter paper, the influence on the mechanical properties is discussed. Also, Firstov et al. ${ }^{11}$ studied the hardness of Ti thin films as a function of the impurity content. Chowdhury et al. ${ }^{12}$ and Takahashi et al. ${ }^{13}$ reported on the influence of impurities on the magnetic properties of thin films. Besides magnetic properties, electrical properties ${ }^{14,15}$ are also affected by the presence of impurities during thin film growth. A number of papers ${ }^{16-20}$ also reports on the change in the intrinsic stress of the thin film as a function of the impurity content. Also, the grain refinement as function of the impurity concentration for Ni was simulated by a kinetic Monte Carlo simulation. ${ }^{21}$ Residual gases and minor leaks are the major source of these contaminants, but also the influence of target impurities on thin film growth has been reported. ${ }^{22}$

The examples given above demonstrate the importance and wide-spread interest of impurities on thin film properties. It is clear that in many cases they are considered to have a degrading impact on the overall quality of the thin film and undesired for many applications. Thin film growth is therefore often studied under ultra high vacuum conditions with base pressures lower than $1 \times 10^{-5} \mathrm{~Pa}$. The deposition rate during magnetron sputter deposition of metals and alloys is in the range of $1 \mathrm{~nm} \mathrm{~s}^{-1}$ or $1 \times 10^{16}$ atoms $\mathrm{cm}^{-2} \mathrm{~s}^{-1}$. The ratio between the impurity and metal impingement fluxes on the substrate, defined as $\tau$, under these conditions is on the order of 0.001. For practical applications, i.e., in industrial vacuum systems, these conditions are often not reached, and the ratio $\tau$ will increase to 0.1 . Even larger values will be obtained when the deposition rate is lowered to reach a higher accuracy in film thickness. In contrast to the many detrimental effects that contaminants can have on the film growth, they can also be used as a tuning mechanism to select the desired thin film properties. For example, Yu and Thompson ${ }^{23}$ have shown that the film stress can be controlled by the impurity content. Another example of a positive effect of impurities has been demonstrated by Gu et al. ${ }^{24}$ for diffusion barrier applications. The improvement of the mechanical properties of high entropy alloys (HEAs) was shown by Braeckman et al. ${ }^{25,26}$ The use of impurities to control the spatial ordering of islands has been demonstrated by Lee and Barabási ${ }^{27}$ and was more recently used to control the growth of graphene by $\mathrm{Wu}$ et $a l^{28}$ It is clear that a deeper understanding of how impurities affect thin film growth-in a beneficial or detrimental way-is of great value. In the current study, the influence of impurities during the film growth of metals and alloys is therefore studied in a range for $\tau$ between 0.01 and 10 or larger. Four single elements systems $(\mathrm{Al}, \mathrm{Ag}, \mathrm{Cr}$, and $\mathrm{Cu}$ ) are studied together with two binary alloys used for the production of E-type thin film thermocouples [chromel $\left(\mathrm{Ni}_{90} \mathrm{Cr}_{10}\right)$ and constantan $\left(\mathrm{Cu}_{55} \mathrm{Ni}_{45}\right)$ ], and one complex alloy also known as a high entropy alloy (HEA) (CoCrCuFeNi).

In more detail, thin films were deposited by magnetron sputtering. The depositions were performed in stainless steel vacuum chambers pumped by a combination of a turbomolecular and a rotary vane pump. The residual gas pressure was lower than $4 \times 10^{-4} \mathrm{~Pa}$ as measured by a Penning gauge. To avoid any influence of the deposition system on the obtained results, the depositions were performed in three different vacuum chambers. A first chamber (volume: $0.021 \mathrm{~m}^{3}$ ) was used for the HEA depositions, a second chamber (volume: 0.052 $\mathrm{m}^{3}$ ) for $\mathrm{Al}, \mathrm{Ni}_{90} \mathrm{Cr}_{10}, \mathrm{Cu}_{55} \mathrm{Ni}_{45}$ depositions, and a third chamber (volume: $0.259 \mathrm{~m}^{3}$ ) for all materials except the HEA. The two inch targets [Testbourne, purity $99.99 \%$ and Plansee Composite Materials $\mathrm{GmbH}$ (CoCrCuFeNi, purity 99.9\%)] were mounted on a home-built magnetron. The magnetron 
was powered with a Hüttinger 1500DC power supply. Experiments were performed at constant argon pressure $(0.3 \mathrm{~Pa})$ as measured with a capacitance gauge. For all materials, a series at constant current of $0.3 \mathrm{~A}$ and $0.6 \mathrm{~A}$ were performed. Only for the high entropy alloy, one series was performed at constant current $(0.3 \mathrm{~A})$ with increasing impurity flux, while another series was deposited at constant impurity flux but with increasing discharge current (range, $0.04 \mathrm{~A}-0.69 \mathrm{~A}$ ) and at an argon pressure of $1 \mathrm{~Pa}$. The films were deposited on RCA cleaned silicon (100) substrates. The film thickness was measured by contact profilometry (Taylor Hobson). For all materials, two series with a film thickness of $100 \mathrm{~nm}$ and $300 \mathrm{~nm}$ were deposited (except the HEA had only one $300 \mathrm{~nm}$ series). It should be remarked that within this experimental range, no influence of the film thickness, discharge current, and argon pressure was observed on the trends discussed in this paper. The film density was determined from the critical angle as measured by X-ray reflectometry (Bruker D8). No systematic trends of the film density as a function of $\tau$ were observed. The metal flux $\mathrm{F}_{M}$ was determined based on the measured thickness, film density, and the deposition time. The deposition rate was approximately $1 \mathrm{~nm} \mathrm{~s}^{-1}$ for all materials, with the exception of $\mathrm{Ag}$ with a deposition rate of $2 \mathrm{~nm} \mathrm{~s}^{-1}$. The impurity flux was controlled according to the following procedure. Before the magnetron discharge was ignited, atmospheric air was leaked into the chamber using a leak valve (Pfeiffer vacuum EVN 116) until a given pressure was reached. Based on the measured pressure, the impingement flux was calculated according to

$$
F_{\text {imp }}=\sum_{i} \frac{z_{i} P_{i}}{\sqrt{2 \pi m_{i} k T}}
$$

where $\mathrm{P}_{i}, \mathrm{z}_{i}$, and $\mathrm{m}_{i}$ refer to the partial pressure, the stoichiometry, and the atomic mass of gas species i, respectively. As atmospheric air was leaked into the chamber, oxygen $(\sim 20 \%, z=2)$ and nitrogen $(\sim 80 \%, z=2)$ were selected as gas species in Eq. (1). When the experiments were performed without additional leaking (HEA series with increasing current), the gas composition as measured with a residual gas analyzer was used in Eq. (1). The main detected species was water. X-ray diffraction (XRD) was performed with a Bruker D8, equipped with a line detector (LynxEye). To avoid any influence of the configuration on the observed trends, one series of samples was measured with a parallel beam configuration (Göbel mirror), while another series was measured in a classical Bragg-Brentano configuration. The instrumental broadening was determined by measuring the XRD pattern for $\mathrm{Cu}$ and $\mathrm{Al}$ powder. The XRD peaks were fitted with a Pearson VII peak shape as described by Ida. ${ }^{29}$ Based on the determined integral breadth, the domain size was calculated according to the Debye-Scherrer equation. No systematic trends in the microstrain could be revealed from the analysis. For all materials and despite the differences in deposition conditions, the same trend in the domain size as a function of the ratio $\tau$ is observed. This is demonstrated for $\mathrm{Ni}_{90} \mathrm{Cr}_{10}$ in Fig. 1. For low values of $\tau$, the domain size remains unaffected in the presence of the impurities. To facilitate the discussion, this regime is indicated as regime I. Consequently, regime II refers to the regime where the domain size becomes a function of $\tau$ (see further). To compare the different elements/conditions, the average value of the domain size in regime I was used to normalize the domain size per material. These measurements are indicated as closed markers in Fig. 1 (and in the figures in the supplementary material). In these figures, the right hand side panel per material depicts the change of the ratio between intensity of the (111) Bragg reflection, and the sum of the intensities of both the (111) and (200) Bragg reflections. All films have a fiber texture, and hence, the change of the intensity ratio is related to a modification of the film texture. For Ag, the texture already changes in regime $\mathrm{I}$. For $\mathrm{Cu}, \mathrm{Al}$, and $\mathrm{Cr}$, no significant trend could be observed over the full range of $\tau$. In the case of the HEA, $\mathrm{Cu}_{55} \mathrm{Ni}_{45}$, and $\mathrm{Cr}_{90} \mathrm{Ni}_{10}$, the texture change
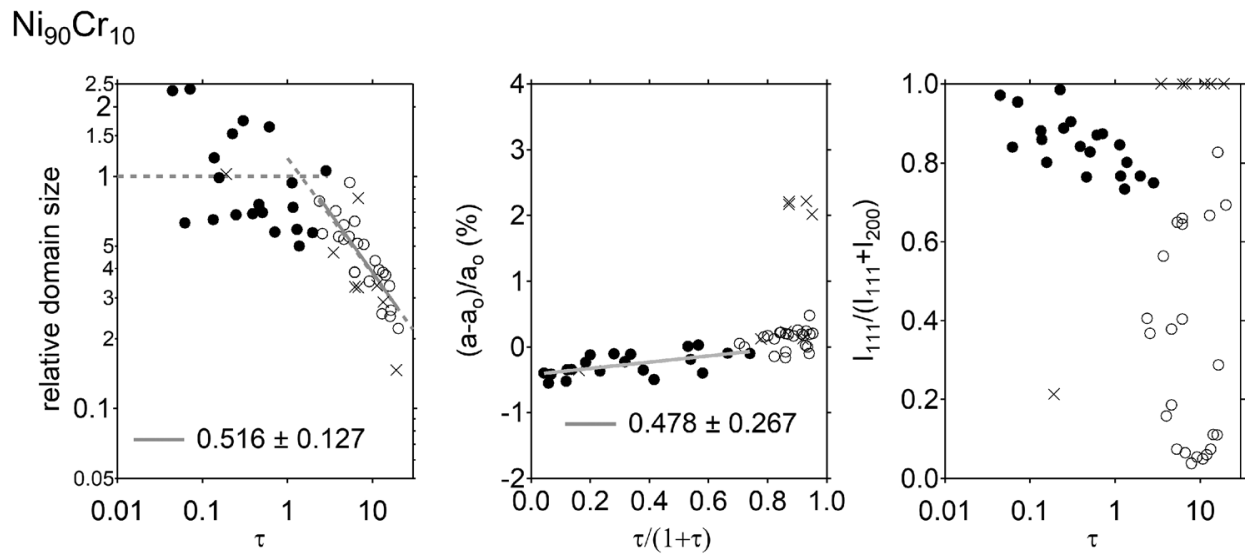

FIG. 1. Overview of the XRD analysis for $\mathrm{Ni}_{90} \mathrm{Cr}_{10}$. Three figures are shown. The left figure presents the relative domain size as a function of ratio $\tau$ between the impurity flux $\mathrm{F}_{\text {imp }}$ and the metal flux $\mathrm{F}_{M}$. The slope [together with its error (95\% confidence interval)] is given. The same $\mathrm{X}$-axis is used for the right figure which presents the ratio between the intensity of the (111) Bragg reflection and the intensity of both the (111) and (200) Bragg reflection. The middle panel shows the relative change of the lattice parameter as a function of the ratio between the impurity flux and the total flux of both the impurities and the metal atoms, i.e., $\tau /(1+\tau)$. Again the slope of the fitted line [together with its error $(95 \%$ confidence interval)] is given. The closed markers refer to measurements where the domain size is not affected by the impurities (relative domain size equals one) while the open markers represents measurements for the regime of the impurity affected growth. The crosses are used for measurements excluded from the analysis. Two main reasons were used to exclude these points. In some cases, the points can be considered as outliners based on a statistical analysis. In other cases, it was not possible to assign them to a specific regime. The data for the other materials are given as supplementary material. 
coincides with the change of the domain size. The out-ofplane orientation of the constituent grains for $\mathrm{Cr}$ was [110] but did not change as a function of $\tau$ (not shown in figure). The different behavior of the studied materials does not allow to generally correlate the domain size with the film texture.

As discussed by $\mathrm{Yu}$ and Thompson, ${ }^{23}$ when the domain (or grain) size remains constant as in regime I, film stress is induced by the incorporation of impurities on metal surfaces and/or on the surface sites of grain boundaries. If the reasoning of these latter authors is followed, the induced stress/ strain is proportional to the impurity concentration, and the ratio between the atomic volume of the impurities and the host metal. The impurity concentration will be proportional to the ratio between the impurity flux $\mathrm{F}_{\text {imp }}$ and the total flux towards the growing film, i.e., $\mathrm{F}_{i m p}+\mathrm{F}_{M}$. The chemical reactivity of the metal will define the final concentration. The impurity incorporation can be evaluated from the increase in the lattice parameter, or stated differently the lattice parameter will increase linearly in regime I as a function of $F_{\text {imp }} /$ $\left(F_{\text {imp }}+F_{M}\right)$. This is valid for all elements except for $\mathrm{Ag}$ (see the supplementary material). The slope is within the error equal to zero, i.e., there is no correlation with the lattice parameter for this latter element. This seems in contrast with the model proposed by $\mathrm{Yu}$ and Thompson. ${ }^{23}$ However, the latter model does not account for the packing fraction of the metal which affects the space available for the incorporation of the impurity within the lattice or at the grain boundary. ${ }^{30}$ This is dealt with by including the octahedral (FCC) or the tetrahedral $(\mathrm{BCC})$ radius. Indeed, the octahedral position within Ag has a similar radius to the atomic radius of oxygen/nitrogen, and therefore, no strain can be induced. As discussed earlier, the chemical reactivity of the element will define the actual concentration of impurities in the thin film, and consequently also the lattice parameter change. The incorporation coefficient depends linearly on the electronegativity difference between the impurity and the host metal as shown by Leroy et al ${ }^{31,32}$ Hence, the measured slope between the lattice parameter and $F_{\text {imp }} /\left(F_{\text {imp }}+F_{M}\right)$ should scale with

$$
\frac{r_{\text {imp }}}{r_{M} c_{\text {void }}} \Delta \epsilon
$$

where $r_{i m p}$ and $r_{M}$ are the radius of the impurities (average weighted value of nitrogen and oxygen atomic radius), and the metal radius (for the alloys an average value was taken), respectively. The electronegativity difference is represented by $\Delta \epsilon$, and $c_{\text {void }}$ is a constant which relates the void size with the metal radius. For FCC lattices $c_{\text {void }}$ equals 0.414 , while for BCC lattices a value of 0.291 is taken. ${ }^{33}$ Figure 2(left) shows that indeed the slope correlates well with Eq. (2). The stronger increase in the lattice parameter in regime II for some materials (see $\mathrm{Cr}, \mathrm{Cu}$, and $\mathrm{Cu}_{55} \mathrm{Ni}_{45}$ ) is more difficult to quantify, as many other processes should be taken into account. For example, although no crystalline oxide or nitride phases have been observed in XRD, the presence of amorphous oxide phases cannot be ruled out.

For 3 out of the 7 materials, i.e., $\mathrm{Al}, \mathrm{Cu}$, and $\mathrm{NiCr}$, the spread on the domain size in regime I is large. No specific correlation between metal properties such as melting temperature and elastic properties, and this high variability has been observed. It is however known that even at room temperature, grain growth can occur for materials such as $\mathrm{Cu}^{34-36}$ and $\mathrm{Al}^{37}$ The lower variability in regime II can probably be explained from the suppression of the driving forces for grain growth as demonstrated by Gianola et $a l^{37}$ In this latter study, specimens deposited at higher base pressures showed no evidence of stress-assisted grain growth. As stated before, in regime II, the domain size becomes function of $\tau$ and can be described by a power law with an exponent $-1 / 2$. The following exponents were found (see the supplementary material): Al: $0.525 \pm 0.154, \mathrm{Cu}: 0.508 \pm 0.088, \mathrm{Cr}$ : $0.512 \pm 0.268, \mathrm{Ag}: 0.393 \pm 0.296, \mathrm{Cu}_{55} \mathrm{Ni}_{45}: 0.507 \pm 0.174$, $\mathrm{Ni}_{90} \mathrm{Cr}_{10}: 0.516 \pm 0.127$, and HEA: $0.494 \pm 0.225$. Note that, although we used Eq. (1) to estimate the contribution of the impurities, the slope on the log-log plot is not affected by the incorporation coefficient ${ }^{31,32}$ of the impurities.

To demonstrate the power law behavior of the domain size in regime II, a kinetic Monte Carlo code was developed. The implementation of this basic model is as follows: Per time step, an amount of adatoms is placed randomly on a square lattice represented by a $2 \mathrm{D}$ zero matrix. This process can be mimicked by switching the state of one or more cells of the 2D square matrix from 0 to 1 . This process represents the deposition flux $\mathrm{F}_{M}$ towards the substrate. During the same time cycle, each adatom, i.e., a cell with a state equal to 1 , is moved randomly over a given number of steps. This represents the adatom diffusion. As the diffusion rate $\mathrm{D}$ is proportional to the number of steps, the ratio between the
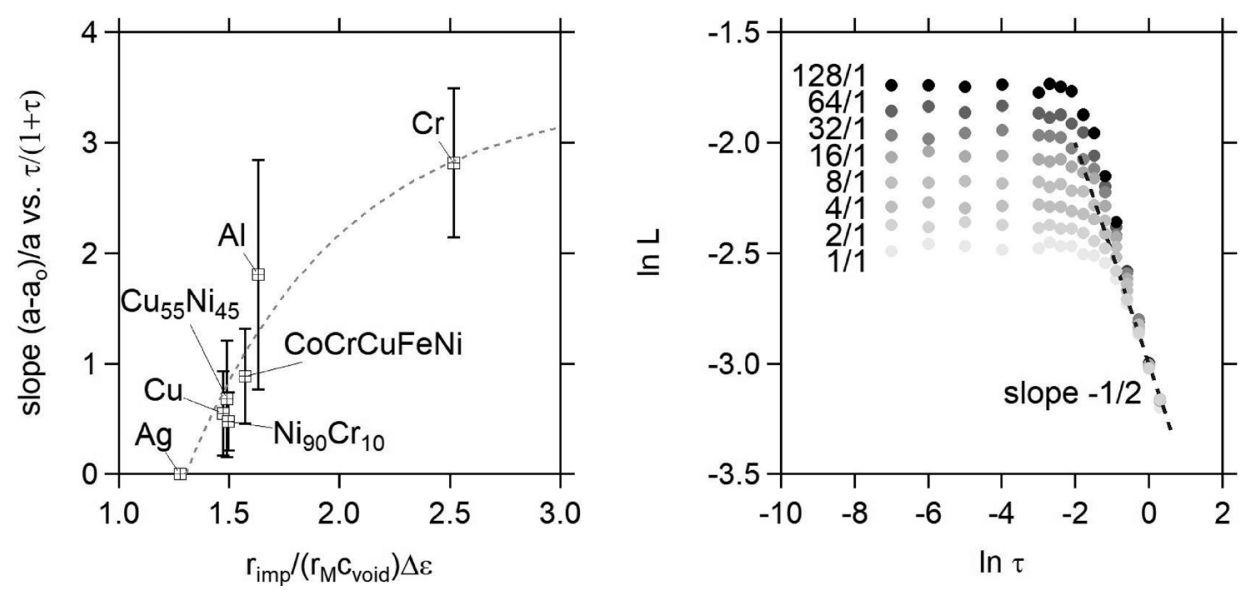

FIG. 2. The left figure shows the slope of the lattice parameter change in regime $\mathrm{I}$ as a function of the parameter derived in Eq. (2). The line is a guide of the eyes. The right figure shows the calculated characteristic length (L) as a function of $\tau$. The different ratios represent to the ratio between the diffusion rate $\mathrm{D}$ and the metal flux $\mathrm{F}_{M}$. 
number of steps and the number of cells filled per cycle is proportional to $\mathrm{D} / \mathrm{F}_{M}$. Depending on the size of the critical nucleus, the program code checks after each diffusion step if sufficient neighboring adatoms are present to form a new stable nucleus, or if the adatom borders to an already existing islands after which the adatom is captured by this island. These two processes modify the state of the representing matrix cell to a value of two. This simulation cycle is repeated until a desired occupation on the substrate surface is reached. As a test, this simple Monte Carlo model should be able to reproduce the results for nucleation theory. ${ }^{38-40}$ According to this latter, the nucleation density scales as a function of $\left(D / F_{M}\right)^{\chi}$, where $\chi$ depends on the critical nucleus size $i^{*}$ as $\chi=i^{*} /\left(i^{*}+2\right)$ for $2 \mathrm{D}$ nucleation. The nucleation density was simulated for $i^{*}=1$ and 2 over a wide range of $\mathrm{D} / \mathrm{F}_{M}$ values. The good agreement between simulations and theory served as a proof for the validity of the code. Although a 2D square lattice has been assumed for reasons of simplicity, this assumption has no effect on the conclusions derived from this model. The characteristic length $\mathrm{L}$ is the mean free path that an adatom can travel before it generates a new nucleus or becomes part of an existing nucleus. The characteristic length relates to the nucleation density as $N^{-2}$. It is assumed that the characteristic length correlates with the grain or domain size. This assumption implies that coalescence in regime $I$ is excluded. The idea of a nucleation-dominated regime without coalescence is based on the good agreement between the calculated characteristic lengths, i.e., in the range of $8-22 \mathrm{~nm}$ for all investigated materials, by the coalescence-free formalism described in the work of Depla and Braeckman ${ }^{46}$ and the observed domain sizes in the impurity-low regime I for all materials. The average domain sizes for the experiments in regime I are in the range of 5-19 nm. In order to simulate the effect of impurities on the nucleation process, the same model structure is preserved with the only difference that the flux $\mathrm{F}$ towards the substrate consists now of a mixture of impurities and adatoms in accordance with $\tau$. After deposition, the impurities are considered immobile, chemisorbed atoms, i.e., they freeze on the surface, and can act as new nucleation centers. Kotrla et $a l .{ }^{41}$ used the same strategy for aluminum in the presence of oxygen impurities. The latter study started from the results of $a b$ initio calculations, indicating that oxygen is essentially immobile on an $\mathrm{Al}(111)$ surface. ${ }^{42}$ Figure 2 (right) shows the results for $\ln (L)$ as a function of $\ln (\tau)$, where the different traces represent different values for $\mathrm{D} / \mathrm{F}_{M}$ (with decreasing values of $\mathrm{D} / \mathrm{F}_{M}$ from top to bottom). For low $\tau$ values, the characteristic length is greatly affected by different values for $\mathrm{D} / \mathrm{F}_{M}$, and increasing the impurity flux seems to have no notable influence on the behavior in this regime. For high $\tau$ values, the characteristic length becomes insensitive to different values of $\mathrm{D} / \mathrm{F}_{M}$ and is therefore independent of the deposition process or the material. In this high contaminated regime, the characteristic length scales as a power law with exponent $-1 / 2$, which is consistent with a nucleation process solely determined by the random distribution of immobile impurity nucleation centers on the surface, i.e., $i^{*} \approx \chi \approx 0$. The results obtained from this basic kinetic Monte Carlo model seem to be in good agreement with the experiments. Indeed, based on the work of Flynn, ${ }^{43}$ the adatom hopping rate for both copper and aluminum can be calculated. At low deposition temperatures, the adatom hopping rate for $\mathrm{Cu}$ is approximately 20 times lower than the value for Al. Nevertheless, the observed behavior in regime II for these two, and all other studied materials, is very similar. Further, the simulated transition point from regime I to regime II hardly depends on the choice of $\mathrm{D} / \mathrm{F}_{M}$, or stated differently, on the choice of the material and the deposition rate. The same behavior is noticed in the experiments. The major exception is $\mathrm{Ag}$, but this can probably be explained from its low chemical reactivity. More elaborated kinetic Monte Carlo models ${ }^{41,44}$ or rate equation models, ${ }^{45}$ with a higher degree of complexity, can be found in literature from which similar conclusions, as presented here, can be drawn. This behavior can be readily understood from the made assumptions. In the model, the impurities are immobile and randomly distributed on the surface. Hence, the average spacing between the impurity atoms decreases as $\tau^{-1 / 2}$. At high values of $\tau$, the characteristic length becomes therefore independent of the material but totally dominated by the impurity spacing. Of course, more research is needed to unravel all possible driving forces that could further contribute to this effect.

In summary, the domain size of seven different materials as function of an increased atmospheric gas impingement flux on the substrate was measured. Small impurity fluxes only have a small effect on the domain size as the characteristic length will be smaller than the capture length based on the distribution of immobile impurities on the surface and therefore the grain size remains mainly unaffected. At higher impurity fluxes, all materials showed a global behavior of a power-law scaling with an exponent of $-1 / 2$ of the domain size as function of the ratio of the impurity to metal impingement flux. This was understood from the perspective of a characteristic length solely determined by the random distribution of nucleated impurities and is consistent with the idea of spontaneous nucleation. A basic kinetic Monte Carlo model was also able to reproduce these results. However, the good agreement with the model should not be wrongly interpreted as an absolute proof that this effect of refinement is inherently related to a nucleation-dominated growth, but rather as an illustration of an underlying geometrical principle of domain size refinement. Therefore, it is not excluded for other models to possibly simulate a similar effect based on different growth principles such as coalescence-hindered growth by incorporation of impurities at the grain boundaries. This general behavior opens the possibility to control and tune the thin film properties by the ratio between the impurity and metal flux.

See supplementary material for the XRD analysis of $\mathrm{Al}$, $\mathrm{Ag}, \mathrm{Cr}, \mathrm{Cu}, \mathrm{Cu}_{55} \mathrm{Ni}_{45}$, and $\mathrm{CoCrCuFeNi}$. The data presented in the supplementary material was obtained in a similar way as for data presented in Fig. 1.

The authors wish to acknowledge the financial support from Ghent University (GOA-ENCLOSE Project) and the Research Foundation Flanders (SPADONA Project).

\footnotetext{
${ }^{1}$ M. Adamik, P. Barna, and I. Tomov, Thin Solid Films 359, 33 (2000).

${ }^{2}$ P. Barna and M. Adamik, Thin Solid Films 317, 27 (1998).

${ }^{3}$ M. Adamik, P. Barna, and I. Tomov, Thin Solid Films 317, 64 (1998).
} 
${ }^{4}$ M. Adamik, I. Tomov, and P. Barna, Solid State Phenom. 56, 213 (1997).

${ }^{5}$ P. Barna, Z. Bodo, Y. Gergely, P. Croce, J. Adam, and P. Jakab, Thin Solid Films 120, 249 (1984).

${ }^{6}$ A. Barna, P. Barna, G. Radnoczi, F. Reicha, and L. Toth, Phys. Status Solidi A 55, 427 (1979).

${ }^{7}$ D. Biro, M. P. Hasaneen, L. Szekely, M. Menyhard, S. Gurban, P. Pekker, I. Dodony, and P. B. Barna, Vacuum 103, 78 (2014).

${ }^{8}$ J. Liu and K. Barmak, Acta Mater. 104, 223 (2016).

${ }^{9}$ S. Mahieu, P. Ghekiere, D. Depla, and R. De Gryse, Thin Solid Films 515, 1229 (2006).

${ }^{10}$ H. Riedl, C. M. Koller, F. Munnik, H. Hutter, F. M. Martin, R. Rachbauer, S. Kolozsvari, M. Bartosik, and P. H. Mayrhofer, Thin Solid Films 603, 39 (2016).

${ }^{11}$ S. Firstov, V. Kulikovsky, T. Rogul, and R. Ctvrtlik, Surf. Coat. Technol. 206, 3580 (2012).

${ }^{12}$ P. Chowdhury, H. C. Barshilia, K. S. Rajam, P. K. Mishra, C. L. Prajapat, and D. V. S. Rao, J. Magn. Magn. Mater. 322, 3266 (2010).

${ }^{13}$ M. Takahashi, M. Tsunoda, and H. Shoji, Vacuum 59, 814 (2000).

${ }^{14}$ A. Canizo-Cabrera, E. Gomez-Barojas, C. Tabares-Munoz, R. SilvaGonzalez, and V. Garcia-Vazquez, Mod. Phys. Lett. B 15, 639 (2001).

${ }^{15}$ S. Brongersma, E. Kerr, I. Vervoort, A. Saerens, and K. Maex, J. Mater. Res. 17, 582-589 (2002).

${ }^{16}$ Y. M. Zhou, Z. Xie, H. N. Xiao, P. F. Hu, and J. He, J. Vac. Sci. Technol., A 27, 109 (2009).

${ }^{17}$ Y. M. Zhou, Z. Xie, H. N. Xiao, P. F. Hu, and J. He, Vacuum 83, 286 (2008).

${ }^{18}$ C. K. Singh, S. Ilango, S. R. Polaki, S. Dash, and A. K. Tyagi, Mater. Res. Express 1, 036401 (2014).

${ }^{19}$ L. Parfitt, O. Karpenko, Z. Rek, S. Yalisove, and J. Bilello, in Thin Films: Stresses and Mechanical Properties VI, Materials Research Society Symposium Proceedings, Vol. 436, edited by W. Gerberich, H. Gao, J. Sundgren, and S. Baker (Materials Research Society, 1996), pp. 505-510.

${ }^{20}$ L. Kendig, Z. Rek, S. Yalisove, and J. Bilello, Surf. Coat. Technol. 132, 124 (2000).

${ }^{21}$ C. C. Battaile and J. J. Hoyt, JOM 57, 71 (2005).

${ }^{22}$ M. Moriyama, T. Morita, S. Tsukimoto, M. Shimada, and M. Murakami, Mater. Trans. 46, 1036 (2005).
${ }^{23}$ H. Z. Yu and C. V. Thompson, J. Vac. Sci. Technol., A 33, 021504 (2015).

${ }^{24}$ G. H. Gu, S. M. Park, and C. G. Park, Met. Mater. Int. 18, 517 (2012).

${ }^{25}$ B. R. Braeckman, F. Misjak, G. Radnoczi, M. Caplovicova, P. H. Djemia, F. Tetard, L. Belliard, and D. Depla, Scr. Mater. 139, 155 (2017).

${ }^{26}$ B. R. Braeckman, P. Djemia, F. Tetard, L. Belliard, and D. Depla, Surf. Coat. Technol. 315, 475 (2017).

${ }^{27}$ C. Lee and A.-L. Barabási, Appl. Phys. Lett. 73, 2651 (1998).

${ }^{28}$ T. Wu, G. Ding, H. Shen, H. Wang, L. Sun, Y. Zhu, D. Jiang, and X. Xie, Nanoscale 5, 5456 (2013).

${ }^{29}$ T. Ida, J. Appl. Crystallogr. 41, 393 (2008).

${ }^{30}$ A. D. Banadaki and S. Patala, npj Comput. Mater. 3, 13 (2017).

${ }^{31}$ W. P. Leroy, S. Mahieu, R. Persoons, and D. Depla, Plasma Processes Polym. 6, S342 (2009).

${ }^{32}$ W. P. Leroy, S. Mahieu, R. Persoons, and D. Depla, Thin Solid Films 518, 1527 (2009).

${ }^{33}$ E. Mittemeijer, Fundamentals of Materials Science: The Microstructure-Property Relationship Using Metals as Model Systems (Springer Berlin Heidelberg, 2010), Chap. 9, p. 405.

${ }^{34}$ C. Detavernier, S. Rossnagel, C. Noyan, S. Guha, C. Cabral, and C. Lavoie, J. Appl. Phys. 94, 2874 (2003).

${ }^{35}$ C. Detavernier, D. Deduytsche, R. Van Meirhaeghe, J. De Baerdemaeker, and C. Dauwe, Appl. Phys. Lett. 82, 1863 (2003).

${ }^{36}$ S. P. Hau-Riege and C. V. Thompson, Appl. Phys. Lett. 76, 309 (2000).

${ }^{37}$ D. Gianola, S. Van Petegem, M. Legros, S. Brandstetter, H. Van Swygenhoven, and K. Hemker, Acta Mater. 54, 2253 (2006).

${ }^{38}$ D. R. Frankl and J. A. Venables, Adv. Phys. 19, 409 (1970).

${ }^{39}$ J. Villain, A. Pimpinelli, L. Tang, and D. Wolf, J. Phys. I 2, 2107 (1992).

${ }^{40}$ T. Michely and J. Krug, Islands, Mounds and Atoms (Springer, Berlin, Heidelberg, 2012).

${ }^{41}$ M. Kotrla, J. Krug, and P. Šmilauer, Surf. Sci. 482-485, 840 (2001).

${ }^{42}$ M. Kotrla, in Proceedings of the 14th Conference of Czech and Slovak Physicists, edited by P. Baroch, M. Kubásek, and Š. Potockỳ (Západočeská Universita, Plzeň, 2002), pp. 369-275.

${ }^{43}$ C. P. Flynn, Phys. Rev. B 71, 085422 (2005).

${ }^{44}$ M. Kotrla, J. Krug, and P. Šmilauer, Phys. Rev. B 62, 2889 (2000).

${ }^{45}$ D. D. Chambliss and K. E. Johnson, Phys. Rev. B 50, 5012 (1994).

${ }^{46}$ D. Depla and B. Braeckman, Thin Solid Films 604, 90 (2016). 